PROCEEDINGS OF THE AMERICAN MATHEMATICAL SOCIETY

Volume 124, Number 11, November 1996

\title{
ON THE SET OF ALL CONTINUOUS FUNCTIONS WITH UNIFORMLY CONVERGENT FOURIER SERIES
}

\author{
HASEO KI
}

(Communicated by Andreas R. Blass)

\begin{abstract}
In this article we calculate the exact location in the Borel hierarchy of $U C F$, the set of all continuous functions on the unit circle with uniformly convergent Fourier series. It turns out to be complete $F_{\sigma \delta}$. Also we prove that any $G_{\delta \sigma}$ set that includes $U C F$ must contain a continuous function with divergent Fourier series.
\end{abstract}

\section{INTRODUCTION}

There are many criteria for uniform convergence of a Fourier series on the unit circle. One can find those tests in $[\mathrm{Zy}]$. In the present paper, we study $U C F$ from the point of view of descriptive set theory. In [Ke], it was a conjecture that $U C F$ is complete $F_{\sigma \delta}$ (i.e., $F_{\sigma \delta}$ but not $G_{\delta \sigma}$ ). A lot of natural complete $F_{\sigma \delta}$ sets have been found. For example, the collection of reals that are normal or simply normal to base $n[\mathrm{KL}] ; C^{\infty}(\mathbb{T})$, the class of infinitely differentiable functions (viewed as a $2 \pi$-periodic function on $\mathbb{R}$ ); and $U C_{X}$, the class of convergent sequences in a separable Banach space $X$, are complete $F_{\sigma \delta}[\mathrm{Ke}]$. It turns out that $U C F$ is complete $F_{\sigma \delta}$. We give two different proofs for it. Ajtai and Kechris [AK] have shown that $E C F$, the set of all continuous functions with everywhere convergent Fourier series, is complete $C A$, i.e., coanalytic non-Borel. We show that there is no $G_{\delta \sigma}$ set $A$ such that $U C F \subseteq A \subseteq E C F$. Hence any $G_{\delta \sigma}$ set that includes $U C F$ must contain a continuous function with divergent Fourier series. From this point of view, although there are many natural complete $F_{\sigma \delta}$ sets, we can claim that $U C F$ is a very interesting set in analysis.

\section{DEFINITIONS AND BACKGROUND}

Let $\mathbb{N}=\{1,2,3, \cdots\}$ be the set of positive integers and $\mathbb{N}^{\mathbb{N}}$ the Polish space with the usual product topology and $\mathbb{N}$ discrete. Let $X$ be a Polish space. A subset $A$ of $X$ is $C A$ if there is a Borel funtion from $\mathbb{N}^{\mathbb{N}}$ to $X$ such that $f\left(\mathbb{N}^{\mathbb{N}}\right)=X-A$. A $C A$ $\left(F_{\sigma \delta}\right)$ subset $A$ of $X$ is called complete $C A\left(F_{\sigma \delta}\right)$ if for any $C A\left(F_{\sigma \delta}\right)$ subset $B$ of $\mathbb{N}^{\mathbb{N}}$, there is a Borel (continuous) function $f$ from $\mathbb{N}^{\mathbb{N}}$ to $X$ such that the preimage of $A$ of $f$ is $B$, i.e., $B=f^{-1}(A)$. From the definition, it is easy to see that no

Received by the editors May 26, 1994 and, in revised form, May 12, 1995.

1991 Mathematics Subject Classification. Primary 04A15, 26A21; Secondary 42 A20.

Key words and phrases. Descriptive set theory, Fourier series, complete $F_{\sigma \delta}$, uniformly convergent Fourier series.

The author was partially supported by GARC-KOSEF. 
complete $C A\left(F_{\sigma \delta}\right)$ set is Borel $\left(G_{\delta \sigma}\right)$. In paticular, if an $F_{\sigma \delta}$ subset $A$ of a Polish space is complete $F_{\sigma \delta}$ and the continuous preimage of an $F_{\sigma \delta}$ subset $B$ of a Polish space, then $B$ is also complete $F_{\sigma \delta}$.

Let $\mathbb{R}$ be the set of real numbers. Let $\mathbb{T}$ denote the unit circle and $I$ the unit interval. Let $E$ be $\mathbb{T}$ or $I$. We denote by $C(E)$ the Polish space of continuous functions on $E$ with the uniform metric

$$
d(f, g)=\sup \{|f(x)-g(x)|: x \in E\} .
$$

$C(\mathbb{T})$ can also be considered as the space of all continuous $2 \pi$-periodic functions on $\mathbb{R}$, viewing $\mathbb{T}$ as $\mathbb{R} / 2 \pi \mathbb{Z}$. Let $U C$ denote the set of all sequences of continuous functions on $I$ that are uniformly convergent, i.e.,

$$
U C=\left\{\left(f_{n}\right) \in C(I)^{\mathbb{N}}:\left(f_{n}\right) \text { converges uniformly }\right\} .
$$

To each $f \in C(\mathbb{T})$, we associate its Fourier series

$$
S[f] \sim \sum_{n=-\infty}^{\infty} \hat{f}(n) e^{i n x}
$$

where $\hat{f}(n)=\frac{1}{2 \pi} \int_{0}^{2 \pi} f(t) e^{-i n t} d t$. Let

$$
S_{n}(f, t)=\sum_{k=-n}^{n} \hat{f}(k) e^{i k t}
$$

be the $n$th partial sum of the Fourier series of $f$. We say the Fourier series of $f$ converges at a point $t \in \mathbb{T}$ if the sequence $\left(S_{n}(f, t)\right)$ converges. Similarly, we define the uniform convergence of the Fourier series of $f$. Let $E C F$ denote the set of all continuous functions with everywhere convergent Fourier series. According to a standard theorem [Ka], the Fourier series of $f$ at $t$ converges to $f(t)$ if it converges. Hence we have

$$
\begin{aligned}
E C F & =\left\{f \in C(\mathbb{T}): \forall t \in[0,2 \pi]\left(\left(S_{n}(f, t)\right) \text { converges }\right)\right\} \\
& =\left\{f \in C(\mathbb{T}): \forall t \in[0,2 \pi]\left(f(t)=\lim _{n \rightarrow \infty} S_{n}(f, t)\right)\right\} .
\end{aligned}
$$

We denote by $N C F$ the complement of $E C F$. Let $U C F$ denote the set all continuous functions with uniformly convergent Fourier series, i.e.,

$$
U C F=\{f \in C(\mathbb{T}): \text { the Fourier series of } f \text { converges uniformly }\} .
$$

\section{RESULTS}

Theorem ([AK]). ECF is complete $C A$.

(See $[\mathrm{AK}]$.)

Proposition 1. $U C F$ and $U C$ are $F_{\sigma \delta}$. 
Proof. Let $\mathbb{Q}$ be the set of all rational numbers. We consider $\mathbb{T}$ as $[0,2 \pi]$ with 0 and $2 \pi$ identified. By the definition of $U C F$,

$$
\begin{aligned}
f \in U C F & \Longleftrightarrow S_{N}(f) \text { converges uniformly } \\
& \Longleftrightarrow \forall a \in \mathbb{N} \exists b \in \mathbb{N} \forall c, d \in \mathbb{N} \forall e \in \mathbb{Q}\left(\left|S_{b+c}(f, e)-S_{b+d}(f, e)\right| \leq \frac{1}{a}\right) \\
& \Longleftrightarrow f \in \bigcap_{a \in \mathbb{N}} \bigcup_{b \in \mathbb{N}} \bigcap_{c, d \in \mathbb{N}} \bigcap_{e \in \mathbb{Q} \cap[0,2 \pi]} V(a, b, c, d, e),
\end{aligned}
$$

where $V(a, b, c, d, e)$ is the collection of $f \in C(\mathbb{T})$ such that $\left|S_{b+c}(f, e)-S_{b+d}(f, e)\right| \leq$ $1 / a$, which is closed, since the function $f \longmapsto \hat{f}(n)$ is continuous. Hence $U F C$ is $F_{\sigma \delta}$. Similarly, so is $U C$. We are done.

Lemma 2. The set $C_{3}=\left\{\alpha \in \mathbb{N}^{\mathbb{N}}: \lim _{n \rightarrow \infty} \alpha(n)=\infty\right\}$ is complete $F_{\sigma \delta}$.

(See $[\mathrm{Ke}, \mathrm{p} .180]$.$) This set will be used to prove our main theorem.$

Proposition 3. $U C$ is complete $F_{\sigma \delta}$.

Proof. We define the function $F$ from $\mathbb{N}^{\mathbb{N}}$ to $C(I)^{\mathbb{N}}$ as follows: for each $\beta \in \mathbb{N}^{\mathbb{N}}$,

$$
F(\beta)=\left(\frac{1}{\beta(n)}\right) \text {. }
$$

Then it is easy to see that

$$
\beta \in C_{3} \Longleftrightarrow F(\beta) \text { converges to } 0 \Longleftrightarrow F(\beta) \text { converges uniformly, }
$$

since $F(\beta)$ is a sequence of constant functions. Clearly, $F$ is continuous. Hence $U C$ is the continuous preimage of $C_{3}$. By Proposition 1 and Lemma 2, $U C$ is complete $F_{\sigma \delta}$.

Theorem 4. There is a continuous function $H$ from $\mathbb{N}^{\mathbb{N}}$ to $C(\mathbb{T})$ such that for all $A$ with $U C F \subseteq A \subseteq E C F$,

$$
\beta \in C_{3} \Longleftrightarrow H(\beta) \in A,
$$

and

$$
\beta \notin C_{3} \Longleftrightarrow H(\beta) \in N C F .
$$

In particular, $U C F$ is complete $F_{\sigma \delta}$.

By this theorem, we have the following corollary.

Corollary 5. There is no $G_{\delta \sigma}$ set $A$ such that

$$
U C F \subseteq A \subseteq E C F,
$$

i.e., any $G_{\delta \sigma}$ set that includes $U C F$ must contain a continuous function with divergent Fourier series.

Proof. Suppose a $G_{\delta \sigma}$ set $A$ satisfies $U C F \subseteq A \subseteq E C F$. Then by Theorem 4, we obtain $H^{-1}(A)=C_{3}$. Since $A$ is $G_{\delta \sigma}$, so is $C_{3}$. By Lemma 2 , this contradicts our assumption. 
It is a basic fact of descriptive set theory [Ke] that any Borel set is coanalytic. Since $E C F$ is complete $C A$ by Theorem $[\mathrm{AK}]$, it is a very natural guess that the complement of $C_{3}$ can be reducible to $E C F-U C F$. In fact, we have the following theorem.

Theorem 6. There is a continuous function $\widetilde{H}$ from $\mathbb{N}^{\mathbb{N}}$ to $C(\mathbb{T})$ such that

$$
\beta \in C_{3} \Longleftrightarrow \widetilde{H}(\beta) \in U C F,
$$

and

$$
\beta \notin C_{3} \Longleftrightarrow \widetilde{H}(\beta) \in E C F-U C F .
$$

In particular, $U C F$ is complete $F_{\sigma \delta}$.

In order to prove Theorem 4 and Theorem 6 , we need the following criterion due to Dini and Lipschitz [Zy, p. 63]. Let $f$ be defined in a closed interval $J$, and let

$$
\omega(\delta)=\omega(\delta ; f)=\sup \{|f(x)-f(y)|: x, y \in J \text { and }|x-y| \leq \delta\} .
$$

The function $\omega(\delta)$ is called the modulus of continuity of $f$.

The Dini-Lipschitz test. If $f$ is continuous and its modulus of continuity $\omega(\delta)$ satisfies the condition $\omega(\delta) \log \delta \rightarrow 0$, then the Fourier series of $f$ converges uniformly.

We introduce the Féjer polynomials, for given $0<n<N \in \mathbb{N}$ and $x \in \mathbb{R}$,

$$
\begin{aligned}
& Q(x, N, n)=2 \sin N x \sum_{k=1}^{n} \frac{\sin k x}{k}, \\
& R(x, N, n)=2 \cos N x \sum_{k=1}^{n} \frac{\sin k x}{k} .
\end{aligned}
$$

These two polynomials were used in $[\mathrm{Zy}]$ to prove that there exists a continuous function whose Fourier series diverges at a point.

Lemma 7. There are positive numbers $C_{1}, C_{2}>0$ such that

$$
|Q|<C_{1} \text { and }|R|<C_{2},
$$

i.e., these polynomials are uniformly bounded in $x, N, n$.

Since

$$
\sum_{k=1}^{n} \frac{\sin k x}{k}
$$

is uniformly bounded in $n$ and $x$, Lemma 7 follows. By Lemma 7, we immediately have the following.

Proposition 8. Let $\left(N_{k}\right)$ and $\left(n_{k}\right)$ be any two sequences of positive integers, with $n_{k}<N_{k}$, and let $\left(\alpha_{k}\right)$ be a sequence of real numbers such that $\alpha_{1}+\alpha_{2}+\alpha_{3}+\cdots<$ $\infty$. Then the series $\sum \alpha_{k} Q\left(x, N_{k}, n_{k}\right), \sum \alpha_{k} R\left(x, N_{k}, n_{k}\right)$ converge to continuous functions. 
Proof of Theorem 4. We fix $A$ with $U C F \subseteq A \subseteq E C F$. Let $\alpha_{k}=2^{-k}, n_{k}=$ $N_{k} / 2=2^{2^{k}}(k=1,2,3, \cdots)$. We define $H$ from $\mathbb{N}^{\mathbb{N}}$ to $C(\mathbb{T})$ as follows: for all $\beta \in \mathbb{N}^{\mathbb{N}}$,

$$
H(\beta)=\sum \alpha_{k} \frac{1}{\beta(k)} Q\left(x, N_{k}, n_{k}\right)
$$

Claim 1. $H$ is continuous and well-defined.

Proof. By Proposition $8, H$ is well-defined. By Lemma 7, it is easy to see that $H$ is continuous.

We divide the rest of proof into two parts so that we have more intuition.

Case 1. $\lim _{n \rightarrow \infty} \beta(k) \neq \infty$.

We want to show that $H(\beta) \in N C F$. For each $k \in \mathbb{N}$ the inequality

$$
\begin{aligned}
& \left|S_{N_{k}+n_{k}}(H(\beta), 0)-S_{N_{k}}(H(\beta), 0)\right|=\left|\sum_{|l| \leq N_{k}+n_{k}} \widehat{H(\beta)}(l)-\sum_{|l| \leq N_{k}} \widehat{H(\beta)}(l)\right| \\
& =\alpha_{k} \frac{1}{\beta(k)}\left(1+\frac{1}{2}+\cdots+\frac{1}{n_{k}}\right)>\alpha_{k} \frac{1}{\beta(k)} \log n_{k}=2^{-k} \frac{1}{\beta(k)} \log 2^{2^{k}} \\
& =\frac{1}{\beta(k)} \log 2
\end{aligned}
$$

holds. Since $\lim _{n \rightarrow \infty} \beta(k) \neq \infty$, there exists a $p \in \mathbb{N}$ such that for infinitely many $k$ 's, $\beta(k)=p$. Hence the Fourier series of $H(\beta)$ does not converge, since in (1) we have $1 / p \log 2$ for infinitely many $k$ 's. So we derive $H(\beta) \in N C F$.

Case 2. $\lim _{n \rightarrow \infty} \beta(k)=\infty$.

We show that $H(\beta) \in U C F$. We will demonstrate that $\omega(\delta ; H(\beta)) \log \delta \rightarrow 0$ as $\delta \rightarrow 0$. Then by the Dini-Lipschitz test, this shows that the Fourier series of $H(\beta)$ converges uniformly. We take any $0<\delta \leq 1 / 2$ and define $\nu=\nu(\delta)$ as the largest integer $k$ satifying $2^{2^{k}} \leq 1 / \delta$ By Lemma 7 , we have the following inequality:

$$
\begin{aligned}
& \left|\sum_{k=\nu+1}^{\infty} \alpha_{k} \frac{1}{\beta(k)} Q\left(x+\delta, N_{k}, n_{k}\right)-\sum_{k=\nu+1}^{\infty} \alpha_{k} \frac{1}{\beta(k)} Q\left(x, N_{k}, n_{k}\right)\right| \\
& \leq 2 C \sum_{k=\nu+1}^{\infty} \alpha_{k} \frac{1}{\beta(k)} \leq 2 C \sup \left\{\frac{1}{\beta(k)}: k>\nu\right\} \sum_{k=\nu+1}^{\infty} \alpha_{k} \\
& =4 C \sup \left\{\frac{1}{\beta(k)}: k>\nu\right\} 2^{-\nu-1} \leq 4 C \sup \left\{\frac{1}{\beta(k)}: k>\nu\right\} \frac{\log 2}{|\log \delta|} .
\end{aligned}
$$

Now we calculate the rest of $H(\beta)$. We clearly have

$$
Q^{\prime}(x, N, n)=N R(x, N, n)+2 \sin N x \sum_{k=1}^{n} \cos k x,\left|Q^{\prime}\right| \leq N C+2 n=n C_{1}
$$


for $N=2 n$ and $C_{1}=2 C+2$. By the mean value theorem, we have the following inequality:

$$
\begin{aligned}
& \left|\sum_{k \leq \nu} \alpha_{k} \frac{1}{\beta(k)} Q\left(x+\delta, N_{k}, n_{k}\right)-\sum_{k \leq \nu} \alpha_{k} \frac{1}{\beta(k)} Q\left(x, N_{k}, n_{k}\right)\right| \\
& \leq C_{1} \delta\left(2^{-1} 2^{2^{1}} \frac{1}{\beta(1)}+\cdots+2^{-\nu} 2^{2^{\nu}} \frac{1}{\beta(\nu)}\right) \\
& \leq C_{1} 2^{2^{-\nu}} \sum_{k \leq \nu} 2^{-k} 2^{2^{k}} \frac{1}{\beta(k)} \leq C_{1} \frac{1}{|\log \delta|} 2^{2^{-\nu}} \sum_{k \leq \nu} 2^{2^{k}-k} \frac{1}{\beta(k)} .
\end{aligned}
$$

By (2) and (3), we have the following:

$$
\text { (4) }|\omega(\delta ; H(\beta)) \log \delta| \leq \max \left\{4 C \sup \left\{\frac{1}{\beta(k)}: k>\nu\right\} \log 2, C_{1} 2^{2^{-\nu}} \sum_{k \leq \nu} 2^{2^{k}-k} \frac{1}{\beta(k)}\right\} \text {. }
$$

Now if $\delta \rightarrow 0$, then $\nu \rightarrow \infty$. So it suffices to show that the right part of (4) goes to 0 as $\nu \rightarrow \infty$. Since $\beta(\nu) \rightarrow \infty$ as $\nu \rightarrow \infty, \sup \{1 / \beta(k): k>\nu\}$ goes to 0 . We need to show that the rest goes to zero as $\nu$ diverges to infinity. This requires the following small claim.

Claim 2. $\sum_{k \leq \nu} 2^{2^{k}-k} \leq 2^{2^{\nu}-\nu+1}$.

Proof. Use induction on $\nu$. For $\nu=1,2^{2-1}=2 \leq 2^{2-1+1}=2^{2}$. Suppose it is true for $\nu$. By the induction assumption, $\sum_{k \leq \nu} 2^{2^{k}-k}+2^{2^{\nu+1}-(\nu+1)} \leq 2^{2^{\nu}-\nu+1}+2^{2^{\nu+1}-(\nu+1)}$. It is enough to show that $2^{2^{\nu}-\nu+1}+2^{2^{\nu+1}}-(\nu+1) \leq 2^{2^{\nu+1}+1}$. Letting $\theta=2^{2^{\nu}}$, one can verify this inequality.

Fix $\epsilon$. Take $N_{0}$ such that $1 / \beta(k)<\epsilon$ for all $k \geq N_{0}$. For this $N_{0}$, we choose $N>N_{0}$ so that $2^{-2^{\nu}+\nu} \sum_{k \leq N_{0}} 2^{2^{k}-k}<\epsilon$ for all $\nu \geq N$. Then for all $\nu \geq N$, by claim 2 , the following inequality is valid:

$$
\begin{aligned}
& 2 C_{1} 2^{-2^{\nu}} 2^{\nu} \sum_{k \leq \nu} 2^{2^{k}-k} \\
& <2 C_{1}\left(2^{-2^{\nu}+\nu} \sum_{k \leq N_{0}} 2^{2^{k}-k} \frac{1}{\beta(k)}+2^{-2^{\nu}+\nu} \sum_{N_{0}<k \leq \nu} 2^{2^{k}-k} \frac{1}{\beta(k)}\right) \\
& <2 C_{1}\left(\epsilon+2^{-2^{\nu}+\nu} \epsilon \sum_{N_{0}<k \leq \nu} 2^{2^{k}-k}\right)<2 \epsilon C_{1}\left(1+\frac{2^{2^{\nu}-\nu+4}}{2^{2^{\nu}-\nu}}\right) \\
& =34 \epsilon C_{1} .
\end{aligned}
$$

Hence the right side of (4) converges to zero as $\nu$ goes to the infinity, i.e., as $\delta \rightarrow 0$. So we derive $H(\beta) \in U C F$.

By case 1 and case 2 , we obtain

$$
\begin{aligned}
& \beta \notin C_{3} \Rightarrow H(\beta) \in N C F \text { and } \\
& \beta \in C_{3} \Rightarrow H(\beta) \in U C F,
\end{aligned}
$$

respectively. Since $N C F$ and $A$ are disjoint, we have the following:

$$
\begin{aligned}
& \beta \notin C_{3} \Longleftrightarrow H(\beta) \in N C F \text { and } \\
& \beta \in C_{3} \Longleftrightarrow H(\beta) \in A .
\end{aligned}
$$


We have shown the first assertion of the theorem. In particular, $C_{3}$ is the preimage of $U C F$. Hence by Lemma 2, the second assertion follows. We have completed the proof of Theorem 4.

Proof of Theorem 6. Instead of $Q$, we use $R$. With $N_{k}, n_{k}$, and $\alpha_{k}$ as in the proof of Theorem 4 , we define $\widetilde{H}$ from $\mathbb{N}^{\mathbb{N}}$ to $C(\mathbb{T})$ as follows: for each $\beta \in \mathbb{N}^{\mathbb{N}}$,

$$
\widetilde{H}(\beta)=\sum \alpha_{k} \frac{1}{\beta(k)} R\left(x, N_{k}, n_{k}\right) .
$$

The same proof as before will demonstrate that this function is well-defined and continuous and that if $\lim _{n \rightarrow \infty} \beta(n)=\infty$, then the Fourier series of $\widetilde{H}(\beta)$ converges uniformly. So it suffices to show that if $\lim _{n \rightarrow \infty} \beta(n) \neq \infty$, then $\widetilde{H}(\beta) \in E C F-$ $U C F$. Suppose $\lim _{n \rightarrow \infty} \beta(n) \neq \infty$. The representation of $\widetilde{H}(\beta)$ as Fourier series is $\sum a_{v} \sin v x$. We see that $\sum a_{v} \sin v x$ converges uniformly for $\delta \leq|x| \leq \pi$ for any $\delta>0$, since the partial sums of $R\left(x, N_{k}, n_{k}\right)$ are uniformly bounded in $k$ and $x$, for $\delta \leq|x| \leq \pi$. The series $\sum a_{v} \sin v x$ contains sines only, and hence it converges for $x=0$, and so everywhere. Now we will show that $\sum a_{v} \sin v x$ does not converge uniformly. It is easy to see that

$$
\sum_{v=1}^{3 n_{k}} a_{v} \sin v x-\sum_{v=1}^{2 n_{k}} a_{v} \sin v x=\sum_{v=2 n_{k}+1}^{3 n_{k}} a_{v} \sin v x=2^{-k} \frac{1}{\beta(k)} \sum_{v=1}^{n_{k}} \frac{\sin \left(2 n_{k}+v\right) x}{v} .
$$

So if we let $x=\pi / 4 n_{k}$, then we have

$$
\left|\sum_{v=1}^{3 n_{k}} a_{v} \sin v x-\sum_{v=1}^{2 n_{k}} a_{v} \sin v x\right|=2^{-k} \frac{1}{\beta(k)} \sum_{v=1}^{n_{k}} \frac{\sin \left(2 n_{k}+v\right) x}{v} \geq 2^{-k} \frac{1}{\beta(k)} \sin \frac{\pi}{4} \sum_{v=1}^{n_{k}} \frac{1}{v},
$$

since, for all $v$ in the interval $1 \leq v \leq n_{k}$,

$$
\frac{3}{4} \pi \geq \frac{\pi}{4 n_{k}}\left(2 n_{k}+v\right) \geq \frac{\pi}{2}
$$

So finally,

$$
\begin{aligned}
\left|\sum_{v=1}^{3 n_{k}} a_{v} \sin v x-\sum_{v=1}^{2 n_{k}} a_{v} \sin v x\right| & \geq 2^{-k} \frac{1}{\beta(k)} \sin \frac{\pi}{4} \sum_{v=1}^{n_{k}} \frac{1}{v} \geq 2^{-k} \frac{1}{\beta(k)} \log n_{k} \sin \frac{\pi}{4} \\
& =\frac{\log 2}{\sqrt{2}} \frac{1}{\beta(k)} .
\end{aligned}
$$

Hence $\sum a_{v} \sin v x$ does not converge uniformly, since in (5) the same value appears for infinitely many $k$ 's. Hence, as in the proof of Theorem 4, we finish the proof of Theorem 6 .

\section{ACKNOWLEDGMENT}

I wish to thank Professor A. Kechris for his constant guidance. The author is indebted to the referee and Professor A. R. Blass for many valuable comments. 


\section{REFERENCES}

[AK] M. Ajtai and A. S. Kechris, The set of continuous functions with everywhere convergent Fourier series, Trans. Amer. Math. Soc. 302 (1987), 207-221. MR 89b:04005

[Ka] Y. Katznelson, An introduction to harmonic analysis, Dover, New York, 1976. MR 54:10976

[Ke] Alexander S. Kechris, Classical Descriptive Set Theory, Springer Verlag, 1995. CMP 95:09

[KL] Haseo Ki and Tom Linton, Normal numbers and subsets of $\mathbb{N}$ with given densities, Fundamenta Mathematicae (2) 144 (1994), 163-179. MR 95e:04005

[Zy] A. Zygmund, Trigonometric series, 2nd ed., Cambridge Univ. Press, 1959. MR 21:6498

Department of Mathematics, California Institute of Technology, Pasadena, CaliFORNIA 91125

Current address: GARC, Department of Mathematics, Seoul National University, Seoul 151742 , Korea

E-mail address: haseo@math.snu.ac.kr 